\title{
Biochemical and Histopathological Correlation in Liver Transplant: The First 180 Days
}

\author{
KeIth S. Henley, ${ }^{1}$ Michael R. LuCey, ${ }^{1}$ HenRy D. ApPelman, ${ }^{2}$ Prabhakar Baliga, ${ }^{3}$ Kimberly A. Brown, ${ }^{1}$ \\ GoRdon D. BURTCH, ${ }^{3}$ DARREll A. CAMPBEll JR., JOHN M. HAM, ${ }^{3}$ ROBERT M. MERION ${ }^{3}$ AND \\ JEREMIAH G. TURCOTTE ${ }^{2}$ \\ The Departments of ${ }^{1}$ Internal Medicine, ${ }^{2}$ Pathology and ${ }^{3}$ Surgery, The University of Michigan Medical School, \\ Ann Arbor, Michigan 48109
}

It is not known whether the histopathology of the liver allograft can be predicted from biochemical measurements in serum with the game confidence as in the native liver. To answer this question we compared the histopathological diagnoses in 170 biopsy specimens from 70 adult transplant recipients obtained during the first 180 days, with the concentrations of the serum bilirubin and the activities of AST, ALT and alkaline phosphatase measured at the same time. The most frequent diagnosis was cholestasis $(n=45)$, which was mild, moderate or severe and which may have been complicated by rejection $(n=28)$ or ischemia ( $=14)$. Hepatitis $(n=14)$, ischemia with rejection $(n=6)$ and spotty focal necrosis $(n=6)$ were diagnosed less frequently. Fifteen biopsy specimens were reported as histopathologically normal. In general, biochemical measurements discriminated poorly between different histopathological diagnoses. The histopathologically normal liver often showed an abnormal pattern of enzymes and an increase in the serum bilirubin level. As a result histopathologically normal biopsy gpecimens were indistinguishable biochemically from those with hepatitis. When two pathological conditions were found to coexist (e.g., cholestasis with either rejection or ischemic necrosis, or ischemic necrosis with rejection), the effect on the serum biochemistry was usually not additive and in some instances returned the biochemical abnormalities toward normal. With the exception of the serum bilirubin level, which increased with the severity of uncomplicated cholestasis, we could not identify a specific pattern of biochemical changes corresponding to a given histopathological diagnosis. We suggest that until more specific noninvasive methods of monitoring the transplanted liver are developed protocol liver biopsies offer the best means of identifying significant pathological conditions in liver allografts. (HEPATOLOGY 1982;16:688-693.)

In 1983 transplantation was recognized as an acceptable treatment for many forms of potentially fatal

Received October 7, 1991; accepted April 15, 1992.

Address reprint requests to: Keith S. Henley, M.D., 3912 Taubman Center, The University of Michigan Medical Center, Ann Arbor, Michigan 48109. 31/1/39443 liver disease (1). In addition to postoperative complications, acute rejection was considered to be a major factor that could prevent a successful outcome (2-4). Rejection was then suspected on the basis of changes in biochemical measurements in serum. These measurements were also used to monitor the response to increased immunosuppression. In these earlier studies, the histopathological findings, when obtained, were considered supportive of the diagnosis. Snover et al. (5) provided a detailed histopathological description of acute cellular rejection consisting of the triad of mixed portal infiltrates, ductitis and endotheliitis. It was also recognized that the clinical suspicion of rejection could not always be confirmed by biopsy specimen. In recognition of this divergence, protocol biopsy specimens have been used routinely in many transplant centers including ours. We have been reluctant to diagnose and treat rejection on the basis of these findings without histopathological documentation and have been surprised to find rejection without any changes in biochemical data. These observations prompted us to ask whether in the transplanted liver a specific relationship exists between the histopathological diagnosis and associated biochemical changes in serum, and whether, therefore, the histopathological interpretation of the allograft can be predicted with the same confidence as in the native liver. We have studied the concentration of the serum bilirubin and the activities in serum of the aminotransferases and alkaline phosphatase because these are known to respond rapidly to changes in liver function.

\section{PATIENTS AND METHODS}

The data from all patients ages 18 years or older who had transplants between March 1, 1989, and April 16, 1990, were analyzed. This coincided with the addition of $3 \mathrm{mmol} / \mathrm{L}$ reduced glutathione to the University of Wisconsin solution in all patients (6) and preceded the initiation of intravenous prostaglandin $E_{1}$ in subsequent patients (7). Data were collected for up to 180 days after transplant.

The protocol provided for liver biopsies to be performed at 7,14 and 21 days postoperatively unless the patient had died or had been discharged or if a biopsy was contraindicated for medical reasons. Additional liver biopsies were performed when clinically indicated. The histopathological diagnoses 


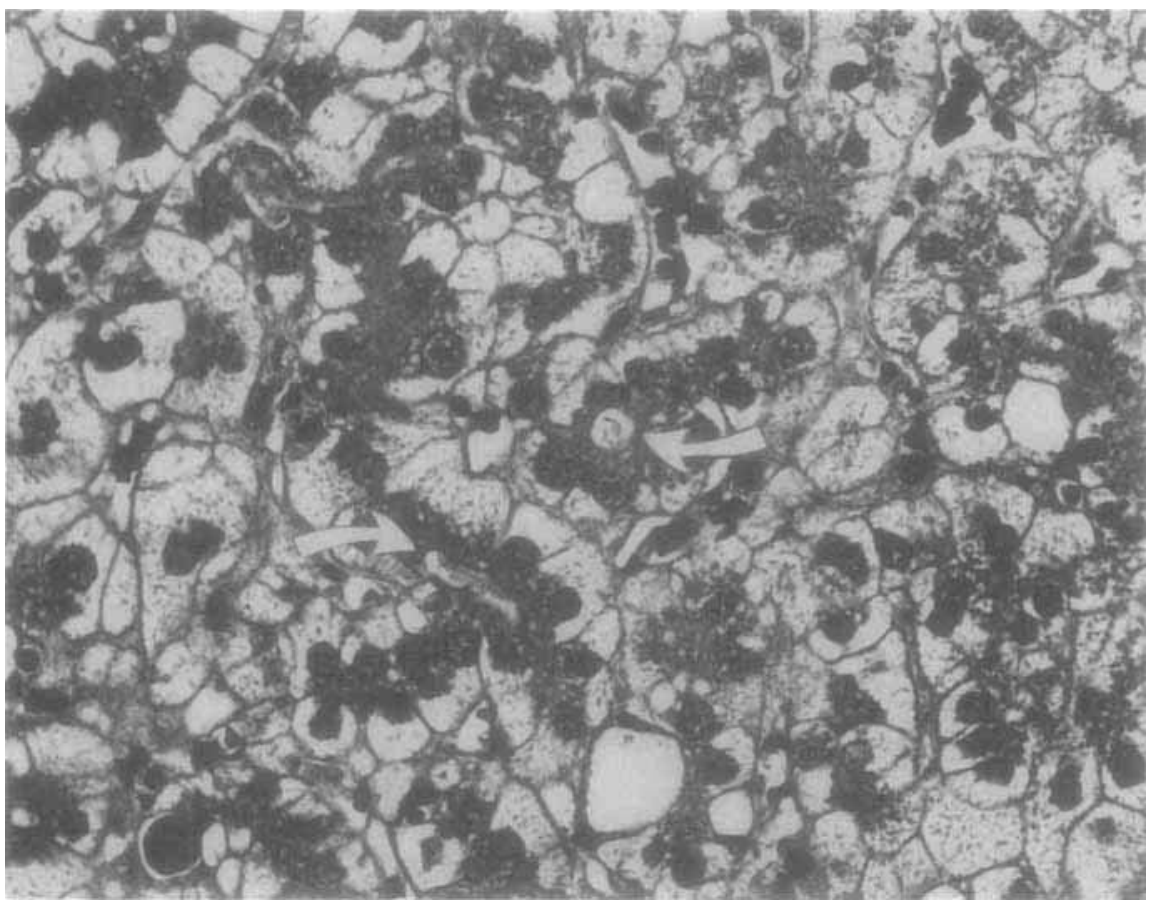

Fig. 1. Cholestasis. The hepatocytes are swollen with rarified cytoplasm that appears finely granular or stringy. Arrows point to two dilated canaliculi containing biliary material. (Original magnification $\times 330$.)

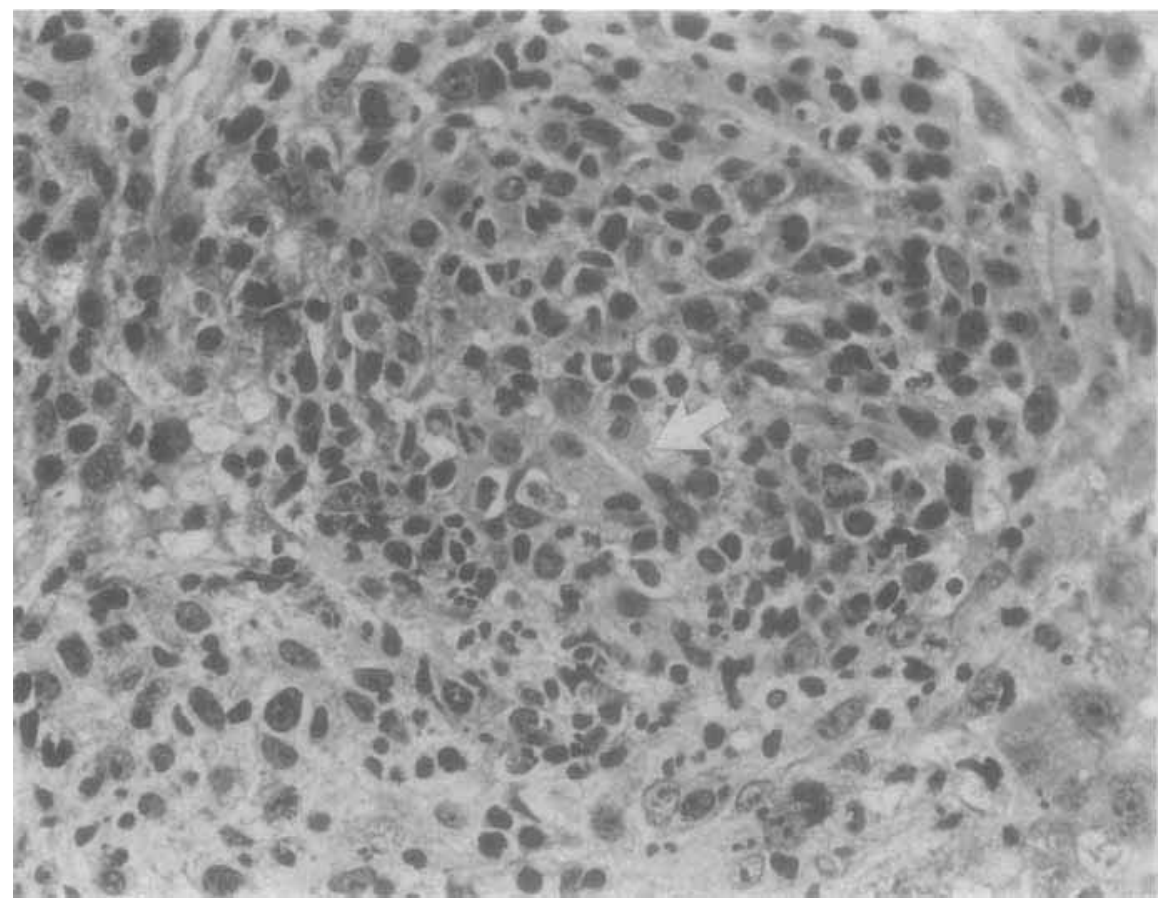

FiG. 2. Acute cellular rejection. This portal tract is expanded by a dense inflammatory cellular infiltrate, many of which are small lymphocytes, but scattered immunoblasts and some neutrophils are present. Arrow points to the remains of a duct that is invaded by lymphocytes and that contains holes in its epithelium. (Original magnification $\times 330$.)

were recorded and compared with the total serum bilirubin level (normal range $=0.2$ to $0.9 \mathrm{mg} / \mathrm{dl}$ ), AST (normal range $=0$ to $45 \mathrm{IU} / \mathrm{L}$ ), ALT (normal range $=0$ to $45 \mathrm{IU} / \mathrm{L}$ ) and alkaline phosphatase (AP) (normal range $=30$ to $130 \mathrm{IU} / \mathrm{L}$ ). In inpatients these diagnoses were almost always obtained just before biopsy, and in a few instances they were obtained on the day before biopsy. In outpatients, laboratory studies were usually obtained less than 24 to $36 \mathrm{hr}$ before the biopsy. When 


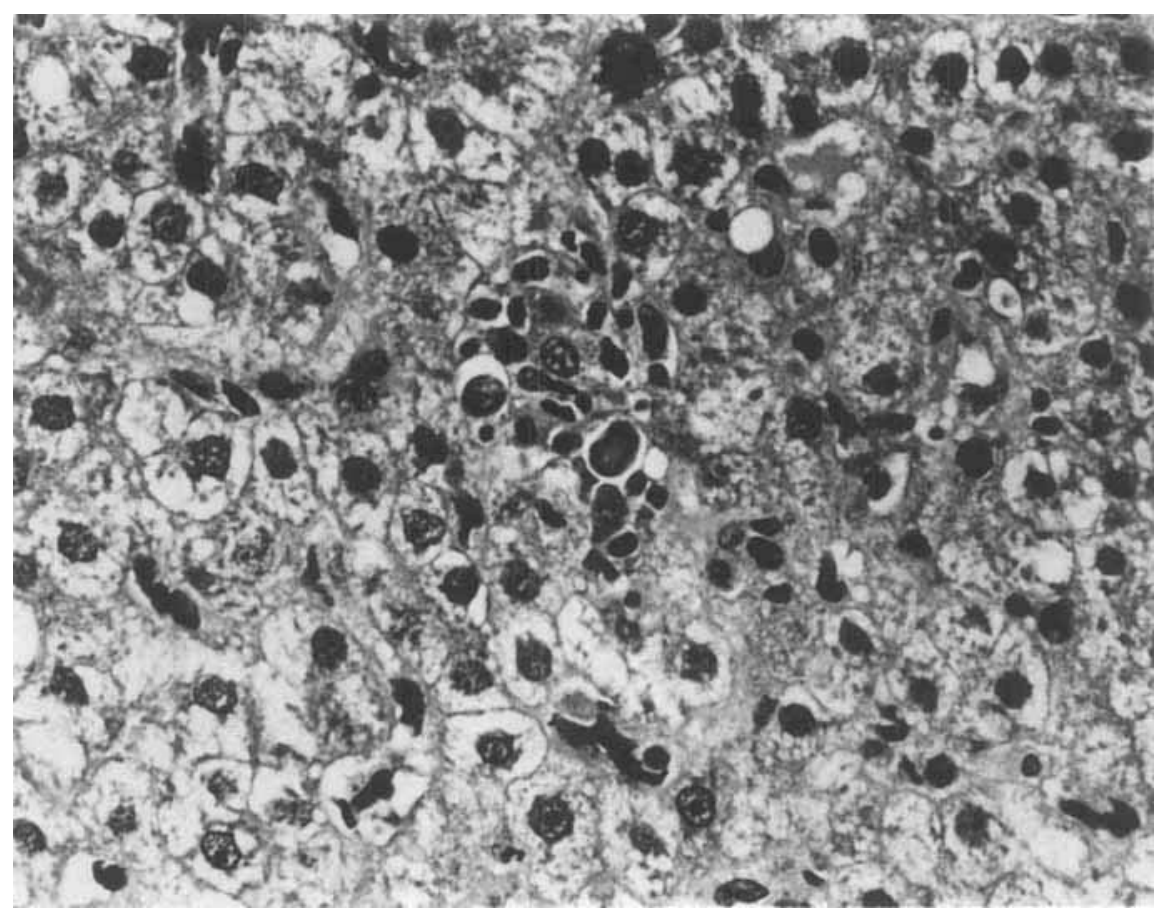

FIG. 3. Spotty lobular necrosis, part of the lobular hepatitis spectrum. In the center of the field is a cluster of lymphocytes that surround three fragments of necrotic hepatocyte cytoplasm seen as the dark rounded blobs. The surrounding hepatocytes are enlarged, and the cord pattern is obliterated. (Original magnification $\times 413$.)

the intervals between laboratory determination and biopsy exceeded $72 \mathrm{hr}$, the biopsy specimen was excluded from analysis.

The postoperative immunosuppression program provided for the administration of azathioprine $(1 \mathrm{mg} / \mathrm{kg} / \mathrm{day})$. On the day of transplant $500 \mathrm{mg}$ of methylprednisone was given, followed by $50 \mathrm{mg}$ of methylprednisone every $6 \mathrm{hr}$ on the first postoperative day. The dose of prednisone was then tapered to $30 \mathrm{mg} /$ day on the $21 \mathrm{st}$ posttransplant day. Minnesota antilymphoblast globulin $(20 \mathrm{mg} / \mathrm{kg} /$ day $)$ was begun on the first postoperative day. When the serum creatinine was less than $3 \mathrm{mg} / \mathrm{dl}$, cyclosporine administration was begun with an initial oral dose of $14 \mathrm{mg} / \mathrm{kg} /$ day and adjusted to a dosage maintenance level of 125 to $150 \mathrm{ng} / \mathrm{ml}$. Minnesota antilymphoblast globulin therapy was stopped after at least 5 days and on the third day of cyclosporine treatment. Patients also received sulfamethoxazole with trimethoprim and acyclovir routinely during the first 30 days.

Histopathological criteria of diagnoses are listed in Table 1.

Statistical analysis was performed by $t$ test. Unless otherwise stated, differences between means when described in the text were statistically significant at $p<0.025$. The study was approved by the Institutional Review Board of the University of Michigan Medical School.

\section{RESULTS}

The analysis was based on 170 biopsy specimens from 70 patients, including 3 patients who required that retransplantation be performed. These three patients underwent their first transplant before April 1, 1989. Another 11 patients could not undergo biopsies because of medical reasons and were therefore excluded from analysis. A total of 113 biopsy specimens were obtained during the first 30 postoperative days. Because the biochemical expression of the different histopathological diagnoses may have changed with the time elapsed since the transplant, the laboratory data from days 1 to 30 in each diagnostic category were compared with those from days 31 to 180 . These were found to be indistinguishable and could therefore be combined. The sole exception was the serum bilirubin level in patients with normal biopsy specimens. This was $2.67 \pm 0.37 \mathrm{mg} / \mathrm{dl}(\mathrm{n}=10)$ for days 1 to 30 and $1.2 \pm 0.2 \mathrm{mg} / \mathrm{dl}(\mathrm{n}=5)$ for days 31 to 180 $(p<0.025)$. Comparison of serum bilirubin values between histopathologically normal and abnormal biopsy specimens was therefore not possible. The total serum bilirubin showed the expected fraction of conjugated bilirubin.

The biochemical data associated with biopsy specimens showing rejection differed from those showing inconclusive evidence of rejection by less than the S.E.M. when the two data were combined. They are therefore presented jointly. In contrast, biopsy specimens showing cholestasis exhibited biochemical patterns that differed according to the degree of histopathological severity and were therefore recorded separately. The laboratory data listed according to the most histopathological diagnoses made most frequently are summarized in Table 2 . Eighteen different diagnoses accounted for the remaining 32 biopsy specimens (including 2 showing rejection and 2 showing incomplete rejection). These were not separately evaluated.

Cholestasis was the most commonly found abnormality and was described as mild in 19 patients, 
moderate in 15 patients and severe in 11 patients. The serum bilirubin level increased with the severity of cholestasis. Levels of AST, although lower in patients showing mild cholestasis, did not discriminate between moderate and severe cholestasis. The intensity of cholestasis was not reflected in the activities of ALT or alkaline phosphatase.

Rejection was the most common accompaniment of cholestasis and occurred in 7,10 and 11 patients with mild, moderate or severe cholestasis, respectively. In patients with mild cholestasis acute cellular rejection resulted in modest increases in the serum bilirubin and aminotransferase values, but the alkaline phosphatase activity remained statistically unchanged when compared with patients whose biopsy specimens showed mild cholestasis without rejection. The effect of rejection on patients with moderate or severe cholestasis was unexpected. A decrease occurred in the activity of AST, and no significant change occurred in the remaining parameters. Rejection complicating ischemic necrosis was seen in six patients, again without significant changes compared with uncomplicated ischemia. Rejection did not modify the biochemical patterns of patients with hepatitis.

Histological appearances suggestive of ischemia were associated with cholestasis in a total of 14 patients. Ischemia lowered the serum bilirubin level among patients with moderate or severe cholestasis, and a similar trend, although statistically not significant, was shown by patients with mild cholestasis. Patients with moderate cholestasis plus ischemia showed an increase in AST. A less marked increase in ALT was seen in patients with mild or moderate cholestasis $(p<0.05)$.

Biochemical studies did not consistently discriminate between different histopathological abnormalities. Thus patients with hepatitis had lower serum bilirubin concentrations than patients with mild moderate or severe cholestasis. The aminotransferase activities were similar except for an increased AST activity in patients with hepatitis compared with those showing mild cholestasis. In contrast the activities of both aminotransferases was lower in patients with hepatitis than in those with moderate or severe cholestasis. These two conditions, however, had their peak incidence on different days. The median date of onset of hepatitis was 100 days after transplant, whereas cholestasis occurred with a median date of onset on the 15th postoperative day.

The enzyme pattern of patients with mild or severe cholestasis was indistinguishable from that of patients with uncomplicated ischemia.

Six patients were reported as showing spotty focal necrosis. This was biochemically indistinguishable from mild cholestasis and could be differentiated from uncomplicated hepatitis only by a modest increase in ALT ( $p<0.05$ ). Ischemia was associated with a higher serum bilirubin level than patients with hepatitis or spotty focal necrosis.

The 15 patients with normal biopsy specimens were often not normal biochemically. Many had increased
TABLE 1. Histopathological criteria for the most frequent diagnoses

\begin{tabular}{cc}
\hline Histopathological Description & Diagnoses \\
\hline Swelling and bile staining of central & Cholestasis
\end{tabular}

zone hepatocytes.

Presence of bilirubin in dilated central zone canaliculi

(Fig. 1)

Periportal and periductal proliferation. Periportal, predominantly neutrophilic infiltrate, Pseudoxanthomas. Intensity of the changes were described as mild, moderate or severe, and if not specified otherwise they were considered to be moderate.

Diagnosed as described by Snover et al. (5). Presence of mixed cellular infiltrate expanding the portal tract with ductitis and endotheliitis. The infiltrate contains lymphocytes, plasma cell, immunoblasts, eosinophils and neutrophils. If one of these criteria was absent (generally ductitis) or modest in extent (generally the infiltrate), the diagnosis was reported as inconclusive for rejection.

Central zone confluent necrosis with collapse with little inflammatory reaction.

Ischemia

This included some biopsy specimens with diffuse hypertrophy of the hepatocytes.

Parenchymal disorganization, necrotic hepatocytes (single or in clusters).

Sinusoidal lymphocytosis, Kupffer cell activation. This is usually accompanied by portal lymphocytosis but not by the mixed portal infiltrate found in rejection.

Patchy necrotic hepatocytes within the lobules including scattered debrisladen phagocytes associated with defects in the hepatocyte cords. Ab-

Acute cellular rejection (Fig. 2) sence of lobular lymphocytosis.

activities of AST transferase and alkaline phosphatase, and most of them had significantly elevated levels of ALT, which increased to an average of more than four times the upper limit of normal. The levels of this enzyme, therefore, did not discriminate between normal patients and patients with hepatitis, cholestasis (regardless of severity), ischemia, or spotty focal necrosis.

To determine whether the difference between patients may have masked a more predictable pattern in the same patients, we examined the records of patients having five or more biopsies. The correlation within patients was not better than between patients. An example is recorded in Table 3.

\section{DISCUSSION}

In the native liver it is usually possible to discriminate between the histopathologically normal and those biopsy 
TABLE 2. Laboratory values by diagnoses ${ }^{a}$

\begin{tabular}{|c|c|c|c|c|}
\hline Diagnoses & Bilirubin (mg/dl) & AST (IU/L) & ALT (IU/L) & $\mathbf{A P}(\mathbf{I U} / \mathbf{L})$ \\
\hline Normal $(n=15)$ & $2.1 \pm 0.30$ & $\begin{array}{c}88 \pm 22 \\
2(3), 4,10\end{array}$ & $225 \pm 60$ & $\begin{array}{c}138 \pm 19 \\
2,(3), 4,10,15\end{array}$ \\
\hline Cholestasis (mild) $(n=19)$ & $\begin{array}{r}4.38 \pm 0.90 \\
3,4,5,(8), 10\end{array}$ & $\begin{array}{c}49 \pm 6.2 \\
3,4,5,6,8,10,1\end{array}$ & $\begin{array}{r}168 \pm 33 \\
(5)(11)\end{array}$ & $\begin{array}{c}269 \pm 42 \\
10,1\end{array}$ \\
\hline Cholestasis (moderate) $(n=15)$ & $\begin{array}{c}10.6 \pm 1.53 \\
2,4,12,8,10,15\end{array}$ & $\begin{array}{l}292 \pm 103 \\
2,12,8,15,1\end{array}$ & $\begin{array}{c}280 \pm 72 \\
8(12)\end{array}$ & $\begin{array}{l}298 \pm 79 \\
\text { (1) }\end{array}$ \\
\hline Cholestasis (severe) $(n=11)$ & $\begin{array}{c}23.5 \pm 4.1 \\
2,3,13,10,15\end{array}$ & $\begin{array}{l}331 \pm 93 \\
2,8,15,1\end{array}$ & $\begin{array}{c}291 \pm 53 \\
8\end{array}$ & $\begin{array}{c}259 \pm 34 \\
1\end{array}$ \\
\hline Cholestasis (moderate) + rejection $(n=10)$ & $7.64 \pm 1.0$ & $\begin{array}{c}77 \pm 11 \\
2\end{array}$ & $253 \pm 38$ & $307 \pm 44$ \\
\hline Cholestasis (severe) + rejection $(n=11)$ & $17.0 \pm 3.3$ & $117 \pm 28$ & $288 \pm 98$ & $240 \pm 48$ \\
\hline Hepatitis $(n=14)$ & $\begin{array}{l}2.34 \pm 0.64 \\
(2), 3,10\end{array}$ & $\begin{array}{c}102 \pm 12.4 \\
\quad 2,3,4\end{array}$ & $\begin{array}{c}134 \pm 21.6 \\
3,4,(15)\end{array}$ & $\begin{array}{l}191 \pm 26 \\
(9), 10\end{array}$ \\
\hline $\begin{array}{l}\text { Cholestasis (mild) }+ \text { ischemic necrosis } \\
(n=4)\end{array}$ & $3.1 \pm 0.47$ & $135 \pm 55$ & $\begin{array}{c}322 \pm 100 \\
(2)\end{array}$ & $182 \pm 34$ \\
\hline $\begin{array}{l}\text { Cholestasis (moderate) }+ \text { ischemic ne- } \\
\text { crosis }(n=5)\end{array}$ & $\begin{array}{c}5.12 \pm 0.80 \\
3\end{array}$ & $\begin{array}{c}690 \pm 99 \\
3\end{array}$ & $777 \pm 418$ & $316 \pm 88$ \\
\hline $\begin{array}{l}\text { Cholestasis (severe) + ischemic necro- } \\
\quad \operatorname{sis}(n=5)\end{array}$ & $\begin{array}{c}11.44 \pm 3.0 \\
4\end{array}$ & $140 \pm 21$ & $267 \pm 67$ & $254 \pm 68$ \\
\hline $\begin{array}{l}\text { Ischemic necrosis }+ \text { rejection }(n=5) \\
\text { Spotty focal necrosis }(n=6)\end{array}$ & $\begin{array}{c}5.47 \pm 1.56 \\
3.48 \pm 0.59 \\
3,4,10\end{array}$ & $\begin{array}{c}208 \pm 90.5 \\
70 \pm 15 \\
3,4,10\end{array}$ & $\begin{array}{c}257 \pm 96 \\
214 \pm 32 \\
(8)\end{array}$ & $\begin{array}{c}484 \pm 148 \\
291 \pm 78 \\
1\end{array}$ \\
\hline
\end{tabular}

$\mathrm{AP}=$ alkaline phosphatase.

${ }^{a}$ Data expressed as mean \pm S.E.M. The diagnoses are listed numerically in the first column on the left. The numbers below the data indicate statistically significant differences $(p<0.025)$ from the mean of the corresponding data in the diagnostic category so identified. For numbers in parentheses the differences are significant at $p<0.05$.

specimens showing hepatitis or cholestasis, with some confidence. Our data shows that in general this does not apply to the transplanted liver during the first 180 postoperative days. In fact, the biochemical patterns shown in Table 2 are more notable for their similarities than for their differences. The only difference between groups that is both statistically significant and sufficiently striking to be clinically helpful is the association between increased levels of the serum bilirubin and the severity of cholestasis. In contrast cholestasis was associated with modest and generally nondiscriminatory increases in the serum alkaline phosphatase. None of the enzymes differentiated the normal from the patient with histological hepatitis.

In the native liver, when two different types of injury are present, the corresponding biochemical pattern would be expected to be more abnormal than when each type of injury was found alone. This was often not true for the transplanted liver. Acute cellular rejection increased the serum bilirubin level only in patients with mild cholestasis, whereas enzyme activities increased little if at all. Acute cellular rejection did not modify the enzyme pattern in patients with hepatitis except for a modest increase in the alkaline phosphatase level, which, however, was statistically indistinguishable from 13 of the 15 conditions listed in Table 2. When cholestasis was associated with ischemic necrosis the biochemical findings were even more surprising because ischemic necrosis appeared to lower the serum bilirubin level. Conversely, if cholestasis were regarded as a complication of ischemic necrosis this complication could not be predicted from measurements in serum. One consequence of these observations is that changes in biochemical measurements could be easily misinterpreted. Thus a fall in the serum bilirubin level in patients with moderate-to-severe cholestasis does not necessarily indicate resolution. It may equally well suggest superimposed rejection (8) or ischemic necrosis.

The unexpected similarities of the biochemical pattern in patients with hepatitis and those whose biopsy specimens were reported as normal is the consequence of two factors: marked enzyme elevations that are sometimes seen in acute hepatitis were not seen here, whereas the patients with normal biopsy specimens showed a range of biochemical abnormalities substantially greater than that usually considered to be 
TABLE 3. Histopathological diagnoses and biochemical data in one patient

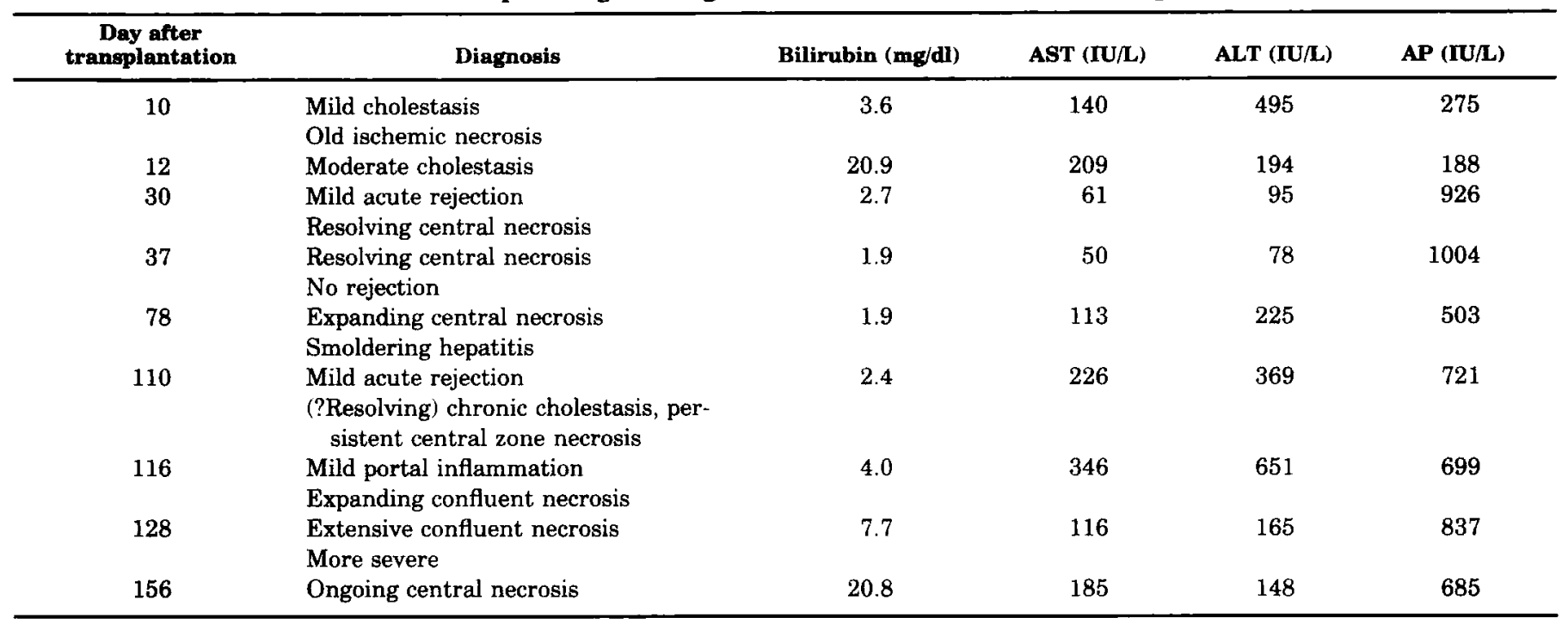

AP = Alkaline phosphatase

normal. The two enzyme patterns therefore converged. One implication of this finding is that routine biochemical studies cannot be relied on to diagnose hepatitis in ongoing or projected studies of the incidence of hepatitis after transplantation.

This study required that patients were sufficiently well compensated to permit a liver biopsy. Data cannot therefore be extended to those patients, often with advanced liver failure, who could not be included in this report. We also could not define the biochemistry of uncomplicated rejection because this was only seen in four patients, and two of these patients had rejections that were reported as incomplete. Our study was also based on the assumption that the liver biopsy is the "gold standard" in the evaluation of the organ after transplantation. Even the normal liver is quite heterogeneous (9), and, for reasons already stated, the transplanted liver may be even more so. Rejection, too, may be found in only one portal triad, whereas adjoining portal triads may be normal. Even with these reservations we suggest that biochemical determinations in the transplanted liver should be interpreted from a new perspective. By implication, newer, noninvasive methods of monitoring liver allografts may need to be developed.

\section{REFERENCES}

1. NIH Consensus Development Conference Statement. Liver transplantation. HEPATOLOGY 1984;4:1075-1095.

2. Grant D, Wall W, Ghent, Duff J, Kutt J, Stiller C, Frei J. Liver transplantation: the problem of rejection. Transplant Proc 1986; 18(suppl 4):163-166.

3. Demetris AJ, Lasky S, Van Thiel DH, Starzl TE, Dekker A. Pathology of hepatic transplantation: a review of 62 cases of adult allograft recipients immunologically suppressed with cyclosporin/steroid regimen. Am J Pathol 1985;118:151-161.

4. Busttil RW, Goldstein LI, Danovitch GM, Ament ME, Memsic LDF Liver transplantation today. Ann Intern Med 1986;104:377-389.

5. Snover DC, Sibley RK, Freese DK, Sharp HL, Bloomer JR, Najarian JS, Ascher NL. Orthotopic liver transplantation: a pathological study of 63 serial liver biopsies from 17 patients with specific reference to the diagnostic features and natural history of rejection. HePaTOLOGY 1984;4:1212-1222.

6. Merion RM, Turcotte JG, Ham JM, Lucey MR, Henley KS, Baliga P, Campbell DA. Early immunological benefits of glutathione supplemented UW solution in hepatic transplantation. Transplant Proc 1991;23:2243-2245.

7. Lucey MR, Brown KA, Merion RM, Nostrant TT, Campbell DA, Turcotte JG, Beresford T. The decreasing incidence of acute allograft rejection: reduced glutathione (GSSH), prostaglandin $\mathbf{E}_{1}$ (PG) or both [Abstract]? Gastroenterology 1991;100:767.

8. Ascher NL, Freese DK, Paradis K, Snover DC, Bloomer JR. Rejection of the transplanted liver. In: Maddrey WC, ed. Transplantation of the liver. New York: Elsevier, 1988:167-190.

9. Gumucio JJ. Hepatocyte heterogeneity: the coming of age. HEPA. TOLOGY 1989;9:154-160. 